\title{
Concussion knowledge and attitudes amongst Stellenbosch University hostel rugby players
}

\author{
W J Kraak, PhD; B A Bernardo, BA; H A Gouws, BSc; A \\ Loubser, BA; I O Vuuren, BSc Hons; M C Coetzee, BSc
}

Department Sport Science, Stellenbosch University, Stellenbosch, South Africa

Corresponding author: WJ Kraak(kjw@sun.ac.za)

Background: Concussion occurs more frequently in contact sports, such as rugby, and is furthermore not fully recognised during play. It is also underreported in the literature, to medical personnel, or to coaches.

Objectives: The objective of this study was to describe the knowledge about and attitudes towards concussion by Stellenbosch University hostel rugby players.

Methods: The study focussed on gathering quantitative information through implementing a cross-sectional study design. One hundred and eighty Stellenbosch University hostel rugby players completed the modified Rosenbaum Concussion Knowledge and Attitudes Survey - Student Version (RoCKAS-ST). The RoCKAS-ST questionnaire is divided into three parts, namely, the evaluation of the Concussion Knowledge Index (CKI) and Concussion Attitudes Index (CAI), and a 16-symptom checklist.

Results: The participants scored on average $75 \%$ in the CKI and $81 \%$ in the CAI. The correlation between CKI and CAI was $\mathrm{r}=0.14$ which is considered a weak positive correlation.

Discussion: The participants demonstrated sufficient knowledge of concussion and thus a safer attitude towards concussion. There were some concerning factors from the knowledge of the concussion questions and the symptoms that may have an effect on attitudes towards concussion.

Conclusion: The study revealed that Stellenbosch University hostel rugby players have sufficient knowledge of what constitutes concussion, as well as the necessity of having safe attitude towards it. However, a small number of participants showed that they still may lack knowledge in certain areas concerning concussion.

Keywords: head injuries; hostel league; university level rugby; survey

\section{S Afr J Sports Med 2018;30:1-5. DOI: 10.17159/2078-516X/2018/v30i1a4404}

Rugby Union is a dynamic, high intensity contact sport which can result in numerous injuries to players. Of these injuries, concussion and other head injuries make up a fair percentage. ${ }^{[1]}$ McCrory et al. defined concussion as a "complex pathophysiological process affecting the brain, which is well presented in sports such as Rugby Union and rugby league". ${ }^{[2]}$ A study by Viljoen indicated that rugby players lacked roughly one-third of the necessary concussion knowledge, which may affect their attitudes towards concussion and increase the risks associated with concussion. [3] Walker found that sub-elite South African rugby players had a fair knowledge of concussion in terms of identification and diagnosis. ${ }^{[4]}$ Players who had a previous history of concussion showed a greater knowledge of this type of injury, thus indicating that knowledge potentially comes from experience rather than from educational programmes. ${ }^{[4]}$

The knowledge of concussion in rugby players can nevertheless have a vital impact on its management and the road to recovery after a player has been concussed. ${ }^{[3]}$ If players have a better understanding of the consequences of concussion and they follow proper protocols, their rate of recovery may drastically improve. [3] Viljoen stated that the players themselves can play an essential role in reducing the incidences of concussion and improving the management thereof. ${ }^{[3]}$ In the same study which evaluated the knowledge and attitudes of concussion by junior amateur high school and senior amateur club rugby players, it was found that these players had insufficient knowledge of concussion and their attitudes/behaviours were deemed unsafe.

Rugby players who understand the severity and effect of concussion and adhere to proper return-to-play guidelines are more likely to have experienced a concussion in rugby themselves and understand the consequences thereof Those who are unaware of the severity of concussion need to be educated on the potential consequences of neglecting specific symptoms. ${ }^{[3,4]}$ To some extent, the players take concussion seriously; however, the mind-set of the players is largely influenced by coaches, fellow teammates and the importance of matches. ${ }^{[4]} \mathrm{O}^{\prime}$ Connell and Molloy concurred stating that 75\% of their participants indicated that they would continue to play in important rugby matches even when concussed. Some of the participants in the study also indicated that they would continue playing with a concussion because they did not want to let their teammates down. ${ }^{[5]}$

When looking at hostel rugby at university level, it can be classified as a social league and the players follow less stringent training regimes compared to those followed in professional and international rugby. ${ }^{[6]}$ The lack of physical conditioning for rugby puts the players at risk of injury during training and matches. These injuries include head injuries, of which concussion was found to be one of the top three types of rugby injuries between 2011 and 2013. ${ }^{[6]}$ The relatively high injury risk of hostel rugby may be as a result of the variability in the skills level of the players and the concurrent academic obligations, which may lead to decreased physical and technical conditioning. Due to their academic responsibilities, the players are often rotated due to unavailability for certain matches. This may lead to a higher risk of injury because of the lack of practice and game time. [7] The teams in hostel rugby consist of university students who have different levels of skill and conditioning. ${ }^{[7]}$ The reasons are that these players do not have sufficient time to attend the gymnasium, technical and field conditioning sessions apart from the games that are played weekly. [6]

In South Africa, the aim of the BokSmart National Rugby Safety Programme is to provide rugby coaches and referees 
with the correct knowledge and technical skills to ensure -that safety and best practice principles are incorporated into all aspects of the game. The BokSmart programme has a section that specifically focusses on concussion and its management. Based on the above background, the objective of this study was to describe the knowledge of and attitudes towards concussion of the Stellenbosch University hostel rugby players.

\section{Methods}

This study focused on gathering quantitative information through the implementation of a cross-sectional study design. Ethical approval (HREC S16_07_129) was obtained from the Research Ethics Committee: Human Research at Stellenbosch University. Hostel rugby players $(n=180)$ from the Stellenbosch University took part in the study. One was excluded because of an incomplete questionnaire and another due to the lack of a consent form. The purpose of the study and the procedures of the questionnaire were explained to the participants. Those who agreed to participate completed an informed consent form and the questionnaire. The participants completed the questionnaires in person enabling them to ask questions or withdraw from the study at any time during the data collection.

The knowledge and attitudes of the participants were measured using a modified Rosenbaum Concussion Knowledge and Attitudes Survey -Student Version (RoCKAS-ST). ${ }^{[3,8]}$ This survey consisted of three main parts, namely, the Concussion Knowledge Index (CKI) , Concussion Attitude Index (CAI) and a 16-symptom checklist. It has five sections, where the 16-symptom checklist may form part of the CKI, but the CKI has its own index. [9] Sections One and Two examined the participants' knowledge of concussion, causes and sequelae. ${ }^{99}$ Section One had 15 true/false questions and Section Two three true/false questions. [9] Questions answered correctly received one point and when answered incorrectly no points. In summary, the scores of Section One, Two and Five were accumulated and the totals ranged from 0-25 to establish the CKI. The participant with a higher score in the CKI revealed a higher level of the knowledge of concussion. The CAI can be used to examine the separate views of all participants. Sections Three and Four consisted of 15 questions, each in a 'Likert Scale' format, ranging from "strongly disagree" to "strongly agree". [9] Out of the 15 questions; five were opinion questions involving the views of the participants and 10 were applied knowledge questions. Section Five of the RoCKAS-ST was replaced with a 16symptom checklist to improve the validity and reliability of the questionnaire. ${ }^{[8]}$ The points received in both sections for each question ranged from one-five points depending on the participant's response to safety. Questions related to safety received one point for a very unsafe response and five points for a very safe response. ${ }^{[9]}$ In summary, the total scores for Sections Three and Four were accumulated and ranged from a total score of 15-75 to establish the CAI score. To determine if the response from the participants' was valid, a validity scale (VS) in Section One of the questionnaire was implemented, with three validation questions in true/false format. A completed questionnaire sheet was regarded as invalid if the participant answered nil or only one question correctly out of those three questions. ${ }^{[9]}$

The data were analysed using Excel Data Analysis. Descriptive statistics for the questions were reported as frequencies and expressed as percentages, means (M), and standard deviations (SD) in order to summarise the score of each index and the participants' questions. The correlation between CKI and CAI was calculated using the Pearson Correlation Coefficient. The Shapiro-Wilk Normality Test was used to test normal distribution of data.

\section{Results}

The correlation between the CKI and CAI was $\mathrm{r}=0.14$ and is considered a weak positive correlation. The CKI average for the hostel rugby players was $18.8 \pm 2.4$. The participants answered $75 \%(18.8 \pm 2.4)$ of the CKI questions correctly. This was calculated by dividing their score by a maximum score of 25 (Tables 4 and 5). Table 1 presents the CKI of the participants, where six of the questions were answered correctly by more than $80 \%$ of the participants. Three of the above-mentioned six questions were answered correctly by more than $90 \%$ of the

Table 1. Statements on concussion evaluating the Concussion Knowledge Index (CKI)

\begin{tabular}{|c|c|c|c|}
\hline & Statements & $\mathbf{n}$ & $\%$ \\
\hline 1. & There is a possible risk of death if a second concussion occurs before the first one has healed. & 151 & 84 \\
\hline 2. & People who had one concussion are more likely to have another concussion. & 135 & 75 \\
\hline 3. & In order to be diagnosed with a concussion, you have to be knocked out. & 177 & 98 \\
\hline 4. & A concussion can only occur if there is a direct hit to the head. & 126 & 70 \\
\hline 5. & Being knocked unconscious always causes permanent damage to the brain. & 146 & 81 \\
\hline 6. & Symptoms of a concussion can last several weeks. & 169 & 94 \\
\hline 7. & Sometimes a second concussion can help a person remember things that were forgotten after the first concussion. & 163 & 91 \\
\hline 8. & $\begin{array}{l}\text { After a concussion occurs, brain imaging (CAT scan, MRI, X-ray etc.) typically shows visible physical damage (bruise, } \\
\text { blood clot) to the brain. }\end{array}$ & 66 & 37 \\
\hline 9. & If you receive one concussion and you have never had a concussion before, you will become less intelligent. & 162 & 90 \\
\hline & After 10 days, symptoms of a concussion are usually completely gone. & 78 & 43 \\
\hline & After a concussion, people can forget who they are and not recognise others but be perfect in every other way. & 70 & 39 \\
\hline & Concussion can sometimes lead to emotional disruptions. & 137 & 76 \\
\hline & An athlete who gets knocked out after getting a concussion is experiencing a coma. & 33 & 18 \\
\hline & There is rarely a risk to long-term health and well-being from multiple concussions. & 138 & 77 \\
\hline
\end{tabular}

$n=$ total number of participants, $\%=$ percentage of respondents who answered correctly 
Table 2. Scenarios on concussion evaluating the CKI

\begin{tabular}{llc}
\multicolumn{1}{c}{ Scenarios } & $\mathbf{n}$ & $\%$ \\
\hline $\begin{array}{l}\text { 1. It is likely that player Q's concussion will affect } \\
\text { his long-term health and well-being. }\end{array}$ & 126 & 70 \\
2. It is likely that player X's concussion will affect & 176 & 98 \\
his long-term health and well-being. & & \\
3. Even though player F is still experiencing the \\
$\begin{array}{l}\text { effects of the concussion, his performance will be } \\
\text { the same as when he had not suffered a } \\
\text { concussion. }\end{array}$
\end{tabular}

Table 3. Concussion symptoms identification ability

\begin{tabular}{lcc}
\hline The following are symptoms of concussion & & \\
\hline Symptoms & $\mathbf{n}$ & $\mathbf{\%}$ \\
Amnesia (memory loss) & 143 & 79 \\
Blurred Vision & 154 & 86 \\
Confusion & 162 & 90 \\
Dizziness & 173 & 96 \\
Headache & 173 & 96 \\
Loss of consciousness & 107 & 59 \\
Nausea & 145 & 81 \\
Sleep disturbances & 93 & 502
\end{tabular}

\begin{tabular}{lcc}
\hline The following are not symptoms of concussion & & \\
\hline Symptoms & $\mathbf{n}$ & $\mathbf{\%}$ \\
Abnormal sense of smell & 7 & 4 \\
Abnormal sense of taste & 12 & 7 \\
Black eye & 38 & 21 \\
Chest Pain & 9 & 5 \\
Nosebleed & 31 & 17 \\
Numbness/Tingling in upper extremity & 40 & 22 \\
Sharp burning pain in the neck & 32 & 18 \\
Weakness of neck range of motion & 79 & 44 \\
\hline
\end{tabular}

$\overline{n=\text { total number of participants, } \%=\text { percentage of respondents who answered }}$ correctly

Table 4. Statements on concussion evaluating the Concussion Attitude Index (CAI) expressed as a percentage

\begin{tabular}{llcc}
\hline \multicolumn{1}{c}{ Statements } & $\begin{array}{c}\text { Safe } \\
(\mathbf{\%})\end{array}$ & $\begin{array}{c}\text { Neutral } \\
(\mathbf{\%})\end{array}$ \\
\hline $\begin{array}{l}\text { I would continue playing a sport while also } \\
\text { having a headache that results from a } \\
\text { concussion }\end{array}$ & 67 & 19 \\
\hline
\end{tabular}

2. I feel that coaches need to be extremely cautious when determining whether an athlete should return to play

3. I feel that concussion are less important than other injuries

$93 \quad 4$

86

869

4. I feel that athletes has a responsibility to return to a game even if it means playing while still experiencing symptoms of a concussion

5. I feel that an athletes who is knocked unconscious be taken to the emergency room

participants. Statement 3 was answered correctly as "false" by $98 \%(n=177)$ of the participants. Statement 6 was correctly answered as "true" by $94 \% \quad(n=169)$ of the participants. Statement 7 was answered correctly as "false" by $91 \%(n=163)$ of the participants. Eight of the statements in Table 1 were answered correctly by less than $80 \%$ of the participants, whereas four of the eight were below $50 \%$. The four most common misconceptions were in Statement 13 which was answered correctly as "true" by only $18 \%(n=33)$ of 154 the participants. Statement 8 was answered correctly as "false" by $37 \%(n=66)$ of the participants. Statement 11 was answered correctly as "false" by $39 \%(n=70)$ of the participants. Statement 10 was answered correctly as "true" by $43 \%(n=78)$ of the participants.

Table 2 indicates that the lowest score achieved for the scenarios was the first question, It is likely that player $Q^{\prime} s$ concussion will affect his long-term health and well-being, 70\% $(n=126)$ of the participants answered correctly. Questions Two and Three had a $98 \%$ and $92 \%$ respectively correct answer rate.

In Table 3, the players correctly identified an average of $80 \%$ of the symptoms $(n=143.75 \pm 27.56)$, whereas an average of $17 \%$ $(n=31)$ identified the incorrect symptoms. Loss of consciousness, 59\% $(n=107)$ and sleep disturbances, 52\% $(n=93)$ were the only correct symptoms that scored below $70 \%$. Both dizziness and headache had the highest score of $96 \%(n=173)$ for the correct symptoms. The highest score for the incorrect symptoms was weakness of the neck's range of motion, which $44 \%(n=79)$ of the players indicated as a symptom.

The CAI average for the hostel rugby players was $81.28 \%$ $(60,98 \pm 6,32)$, this being which was calculated by dividing their score by a maximum of 75 (Tables 4 and 5). Hostel rugby players on average answered $81 \%$ of the CAI questions correctly. A Likert scale was used with a score out of five, where four-five was classified as "safe", three as "neutral" and onetwo as "unsafe". Table 4 had an average score of $84 \%$ that was scored as safe and $10 \%$ that was scored as neutral with CAI. In Table $4,67 \%$ of the participants had a "safe" response where $19 \%$ was "neutral" for Question 1. In Question 2, only $4 \%$ of the participants gave a "neutral" answer, which was the lowest, and $93 \%$ had a "safe" answer, which was the highest.

The scenario with the lowest "safe" percentage of $58 \%$, where most players felt that the athlete who suffered a concussion during the semi-final playoff game should have returned to play during the same game. In another scenario, only $60 \%$ of players scored "safe" and it was stated that athletes would feel that the trainer rather than the athlete who suffered a concussion should make the decision about returning athlete to play. Comparing the scenario of the player that was concussed during the semi-final game, it was shown that most athletes felt that the athlete who sustained a concussion during the first game of the season should have returned to play during this game and that there was a $21 \%$ difference between the scenarios. The first game of the season also had a "safe" score of $79 \%$.

\section{Discussion}

The aim of the study was to evaluate and describe the knowledge and attitudes of Stellenbosch University hostel rugby players to concussion. The major findings of the study indicated a weak positive correlation $(\mathrm{r}=0.14)$ between $\mathrm{CKI}$ and CAI. A weak correlation is 0.1 to 0.3 and a strong correlation is 1.0 to 0.5 . A weak correlation means that as the CKI increases or decreases, there is a lower possibility of there being a relationship with the CAI. The CKI mean score achieved by the 
Table 5. Scenarios on concussion evaluating the Concussion Attitude Index (CAI) expressed as a percentage

\begin{tabular}{|c|c|c|}
\hline Scenarios & $\begin{array}{l}\text { Safe } \\
(\%)\end{array}$ & $\begin{array}{c}\text { Neutral } \\
(\%)\end{array}$ \\
\hline $\begin{array}{l}\text { 1. I feel that Coach A made the right decision to } \\
\text { keep Player R out of the game. }\end{array}$ & 94 & 3 \\
\hline $\begin{array}{l}\text { 2. Most athletes would feel that Coach A made } \\
\text { the right decision to keep Player } \mathrm{R} \text { out of the }\end{array}$ & 70 & 16 \\
\hline
\end{tabular}

3. I feel that Athlete $\mathrm{M}$ should have returned to play during the first game of the season (that same game of the injury).

4. Most athletes would feel that Athlete M should have returned to play during the first game of the season (that same game of the injury).

5. I feel that Athlete $\mathrm{O}$ should have returned to play during the semi-final playoff game.

6. Most athletes feel that Athlete $\mathrm{O}$ should have returned to play during the semi-final playoff game.

7. I feel that the athletic trainer rather than Athlete $\mathrm{R}$ should make the decision about Athlete $\mathrm{R}$ returning to play.

8. Most athletes would feel that the athletic trainer rather than Athlete $\mathrm{R}$ should make the decision about returning Athlete $\mathrm{R}$ to play.

9. I feel that Athlete $\mathrm{H}$ should tell coach about the symptoms.

10. Most athletes would feel that Athlete $\mathrm{H}$ should tell coach about the symptoms.

participants was $18.78 \pm 2.44$ out of a maximum score of 25 . The participants, on average, answered $75 \%$ of the CKI questions correctly. Based on the results of this study, it was found that the participants had a basic knowledge of concussion. In Section One of the questionnaire, over $50 \%$ of the participants lacked the knowledge of concussion in four questions. This is similar to a previous study done by Viljoen, where three of those questions had very similar results ( see Table 1 Statement 8 and Statement 11). ${ }^{[3]}$ This shows that there are areas of concern and misconceptions regarding concussion.

In Table 3 where participants had to identify the correct symptoms, $59 \%$ identified loss of consciousness and 52\% indicated sleep disturbances as a symptom. In studies by Hecimovich and Viljoen sleep disturbances were shown as the lowest correctly identified symptom. [3,10] However, having sufficient knowledge of concussion is not enough, as concussion is a serious matter in sport, specifically rugby. Kaut et al. found that the focus should shift from knowledge of concussion to behavioural responses following such injuries. ${ }^{[3]}$ Viljoen stated that rugby players can play a crucial part in reducing the incidence of concussion and in improving the management of concussed players. ${ }^{[3]}$ In the Viljoen study on the knowledge of concussion in junior amateur high school players and senior club players it was shown that players lacked knowledge of concussion and that their attitudes and behaviours towards concussion were unsafe. [3] The CAI average for the participants tested was $60.98 \pm 6.34$ with a maximum score of 75 . On average, the participants answered
$81 \%$ of the CAI questions correctly. This shows that the participants have a good, safe attitude toward concussion but are influenced by parents, teammates, coaches and the importance of specific matches. The above-mentioned statement is supported by studies done by $\mathrm{O}^{\prime}$ Connell and Molloy and Delahunty et al., in which respectively $75 \%$ and $73 \%$ of the players indicated that they would play with a concussion in important matches. ${ }^{[5,11]}$ The main reason for wanting to continue was the fear of letting their teammates down. Although these factors play a role, a player with a good concussion attitude may still choose to act in an incorrect or unsafe manner following concussion without being influenced.

The current study revealed that $58 \%$ of the players deemed that playing with a concussion (see Table 5 Statement 6) as "safe". If this is compared with another scenario in the questionnaire, it was found that if it was the first game of the season, as opposed to a playoff game, $79 \%$ of the players felt that the player should not return to the game. These findings support the claims in the study by O'Connell and Molloy namely, that players are influenced by important matches. ${ }^{[5]}$ Viljoen highlights that there needs to be educational workshops on concussion conducted specifically for rugby players. [3] Compared to the study done by Viljoen,[3] this present study has shown superior results with regards to concussion knowledge and attitudes. The above-mentioned could be as a result of increased awareness of the dangers of concussion, as well as improved management by coaches and clubs.

\section{Conclusion}

In conclusion, the results in this study indicated that Stellenbosch University hostel rugby players have sufficient knowledge of what constitutes concussion and a good attitude towards the condition. However, a small number of the players clearly lacked knowledge in certain vital areas of concussion, such as whether a player should play in an important rugby match if suffering from concussion. These vital areas might play an important role in the behaviour of rugby players who sustained the concussion, in terms of avoiding the potential risks of concussion, such as brain damage or even death. For future studies related to concussion, researchers should take note that testing the knowledge and attitudes of concussion on rugby players may be challenging, because there might be a difference between answering a questionnaire and the actual behaviour of players during a match or practice session when referring to concussion. A limitation of the current study was that the content of the questionnaire could have been shared by the different participants thus influencing the other participants in the study.

\section{References}

1. Gardner AJ, Iverson GL, Williams WH, et al. A systematic review and meta-analysis of concussion in rugby union. Sports Med 2014;44(12):1717-1731. [doi: 10.1007/s40279-014-0233-3]

2. McCrory P, Meeuwisse WH, Aubry M, et al. Consensus statement on Concussion in Sport- the 4th International Conference on Concussion in Sport held in Zurich, November 2012. J Sci Med Sport 2013;16(3):178-189. [doi: 10.1016/j.jsams.2013.02.009] 
3. Viljoen CT, M Schoemman, C Brandt, et al.. Concussion knowledge and attitudes among amateur South African rugby players. S Afr J Sports Med 2017;29(1):1-6. [doi: 10.17159/2413-3108/2015/v27i2a492]

4. Walker SP. Concussion knowledge and return-to-play attitudes among subelite rugby union players. S Afr J Sports Med 2015;27(2):50-54. [doi: 10.7196/SAJSM.536]

5. O'Connell E, Molloy MG. Concussion in rugby: knowledge and attitudes of players. Ir J Med Sci 2016;185(2):521-528. [doi: 10.1007/s11845-015-1313-6]

6. Barret A. The epidemiology of hostel rugby injuries at Stellenbosch University. Unpublished MSc (Sport Science) thesis. Stellenbosch: Stellenbosch University, 2015.

7. Mathewson E, Grobbelaar R. Tackle-injury epidemiology in koshuis rugby players at Stellenbosch University. S Afr J Sports Med 2015;27(3):72-75. [ doi:10.7196/SAJSM.8091]

8. Williams J. Concussion knowledge and attitudes in English football (soccer). Unpublished M.S. (Kinesiology). Georgia: Georgia Southern University, 2013.
9. Rosenbaum AM, Arnett PA. The development of a survey to examine knowledge about and attitudes toward concussion in high-school students. J Clin Exp Neuropsychol 2010;32(1):4455. [doi: 10.1080/13803390902806535]

10. Hecimovich M, King D, Marais I. Player and parent concussion knowledge and awareness in youth Australian Rules Football. The Sport Journal 2016. http://thesportjournal.org/article/player-and-parentconcussion-knowledge-and-awareness-in-youth-australianrules-football/. [Accessed 1 June 2017]

11. Delahunty SP, Delahunt E, Condon B, et al. Prevalence of and attitudes about concussion in Irish schools' rugby union players. J Sch Health 2015;85(1):17-26. [doi: 10.1111/josh.12219]

12. Kaut KP, DePompei R, Kerr J, et al. Reports of head injury and symptom knowledge among college athletes: implications for assessment and educational intervention. Clin J Sport Med 2003:13(4):213-221 